\title{
Initial In-Vivo Analysis of 3D Heterogeneous Brain Computations for Model-Updated Image-Guided Neurosurgery
}

\author{
Michael Miga ${ }^{1}$, Keith Paulsen ${ }^{1,2,3}$, Francis Kennedy ${ }^{1}$, Jack Hoopes ${ }^{1,2,3}$, \\ Alex Hartov ${ }^{1,2}$, and David Roberts ${ }^{2,3}$ \\ 1 Dartmouth College, Thayer School of Engineering, HB8000, Hanover, NH 03755 \\ \{michael.miga, keith.paulsen, francis.kennedy, p.jack.hoopes, \\ alex.hartov, david.w.roberts \}@dartmouth.edu \\ http://ww. thayer.dartmouth. edu/thayer/ \\ 2 Dartmouth Hitchcock Medical Center, Lebanon, NH 03756 \\ ${ }^{3}$ Norris Cotton Cancer Center, Lebanon, NH, 03756
}

\begin{abstract}
Registration error resulting from intraoperative brain shift due to applied surgical loads has long been recognized as one of the most challenging problems in the field of frameless stereotactic neurosurgery. To address this problem, we have developed a 3-dimensional finite element model of the brain and have begun to quantify its predictive capability in an in vivo porcine model. Previous studies have shown that we can predict the average total displacement within $15 \%$ and $6.6 \%$ error using intraparenchymal and temporal deformation sources, respectively, under relatively simple model assumptions. In this paper, we present preliminary results using a heterogeneous model with an expanding temporally located mass and show that we are capable of predicting an average total displacement to $5.7 \%$ under similar model initial and boundary conditions. We also demonstrate that our approach can be viewed as having the capability of recapturing approximately $75 \%$ of the registration inaccuracy that may be generated by preoperative-based image-guided neurosurgery.
\end{abstract}

\section{Introduction}

Current state-of-the-art image-guided surgery involves coregistration of the patient, the operating room coordinate space, patient-specific images generated by CT (computed tomographic) or MR (magnetic resonance), and surgical instrument location [1], [2]. This capability has dramatically impacted the field of frameless stereotactic neurosurgery by allowing detailed navigational information to be monitored in real-time during surgery. However, often during a neurosurgical procedure, tissue is purposely retracted/resected which causes a subsequent shift of the cortical surface as well as subsurface structures. Roberts et. al. performed a detailed study tracking cortical surface shift over 28 surgeries involving a variety of neurosurgical procedures and found on average a $1 \mathrm{~cm}$ shift of the surface with a definite predisposition in the direction of gravity [3]. Hill 
et. al. performed a comparable study on 5 cases and evaluated overlays of the deformed surface on the original MR to understand the nature of the intraoperative brain shift. They found surface shifts that ranged from $0.3-7.4 \mathrm{~mm}$ and that the surface tended to sink in the direction of gravity[4]. Dickhaus et. al. performed a 10 patient study with only tumor resections but used intraoperative MR which allowed quantification of subsurface movement. They found that surface shift was on the order of $2 \mathrm{~cm}$, while subsurface movement was 6 to $7 \mathrm{~mm}$ for positions located at the interhemispheric fissure and the lateral ventricles [5].

This data suggests that coregistered image studies performed preoperatively cannot accurately account for such shift which in turn could lead to surgical error when relied upon for navigational information. To date, the only solutions to account for this shift have come in the form of intraoperative CT/MR which is costly and cumbersome and intraoperative ultrasound which provides a limited database with significantly lower contrast resolution. We have elected to pursue a physics-based modeling approach to account for intraoperative tissue deformation. Using the finite element method (FEM), we have modeled the brain using porous-media physics and have begun to quantify computational model accuracy using an in vivo porcine system in which we impart simulated surgical loads [6]. Previous studies have shown we can produce an average total displacement with errors less than $15 \%$ for an intraparenchymal placement of the deformation source [7], and less than $6.6 \%$ for a temporally located source under assumptions of homogeneous tissue properties [8]. In this paper, we have modeled the temporal mass expansion with added tissue heterogeneity, specifically gray/white matter delineation, in order to investigate the extent to which this increase in model complexity improves the data-model match.

\section{Model}

The theory used to model the brain originates from the soil mechanics literature and has been used in the context of soil consolidation. Deformation by consolidation theory [9] is characterized by an instantaneous deformation at the load contact area followed by subsequent additional displacement over time as the interstitial fluid drains in the direction of strain-induced pressure gradients (i.e. from high to low pressure). This theory characterizes brain tissue as a biphasic system consisting of a solid matrix and an interstitial fluid. The solid matrix is isotropic and follows the laws of linear elasticity. The fluid component represented by a pressure distribution flows in accordance with Darcy's law and is usually considered incompressible. The governing equation describing mechanical equilibrium is,

$$
\nabla \cdot G \nabla \mathbf{u}+\frac{G}{1-2 \nu} \nabla \varepsilon-\alpha \nabla p=0 .
$$

where $G$ is the shear modulus $\left(G=\frac{E}{2(1+\nu)}\right), \nu$ is Poisson's ratio, $\alpha$ is the ratio of fluid volume extracted to volume change of tissue under compression, $\epsilon$ is the volumetric strain $(\epsilon=\nabla \cdot \mathbf{u}), \mathbf{u}$ is the displacement vector, and $p$ is the interstitial 
pressure. In order to complete the description, a constitutive equation relating volumetric strain to fluid drainage is represented as

$$
\nabla \cdot k \nabla p-\alpha \frac{\partial \varepsilon}{\partial t}-\frac{1}{S} \frac{\partial p}{\partial t}=0,
$$

where $k$ is the hydraulic conductivity, and $1 \backslash S$ is a void compressibility constant. In the literature, the fluid component of the model is typically considered incompressible for brain tissue, which we have also adopted (i.e. $\frac{1}{S}=0, \alpha=1$ ). The weighted residual form of the above equations along with a stability analysis has been presented previously [7], [10]. The method of solution used is a fully implicit Galerkin finite element scheme.

Unfortunately, only a modest amount of data on brain tissue mechanical properties has been reported in the literature. Early studies used mechanical devices to measure properties in vivo but were semi-quantitative at best [11]. A new and exciting area of research has emerged using MR and ultrasound to image transverse strain waves thereby allowing the calculation of regional mechanical properties based on strain measurements [12]. While more quantitative tissue property information can be anticipated in the future based on these newer techniques, the current uncertainty in brain tissue mechanical properties has led us to investigate the effect of varying Young's modulus and Poisson's ratio in our simplified homogenous model as well as the pressure gradient which acts as a distributed body force with respect to elastic deformation [13]. The results indicate that an optimal Young's modulus exists which varies with Poisson's ratio. The calculations also indicate that an optimal pressure gradient for a given Young's modulus and Poisson's ratio exists as well.

Based on previous results and the few literature citations on brain tissue properties which exist, we have performed a set of six calculations with different combinations of tissue properties as reported in Section 4. For comparison purposes three of these simulations use our homogeneous model with the elastic properties taken from our previous experience [13]. In addition, two different values of hydraulic conductivity have been assumed which span the maximum and minimum values in [14], [15]. In the three heterogeneous calculations, tissue property parameters have been taken from Nagashima et. al. [14] and Basser [15]. The Nagashima data is based on the feline brain for moduli whereas Basser performed analytical work to deduce his property values.

\section{Methods}

In conjunction with the realization of a numerical model, we have developed a method of quantification using an in vivo porcine experimental protocol. Prior to surgical procedures, a detailed MR series is taken of the pig cranium. These scans guide the FEM discretization process. Using ANALYZE Version 7.5 - Biomedical Imaging Resource (Mayo Foundation, Rochester, M.N.), we segment the 3D volume of interest and execute a discrete marching cubes allgorithm to render a discretized description of the surface [16]. We then produce 
a tetrahedral grid on the interior of the domain defined by this boundary using customized mesh generation software [17]. Following the generation of the volumetric grid, we proceed to derive element tissue types by examining the average intensity value over an element based on the original MR image voxels thus creating a heterogeneous model. In this paper, we used thresholding to classify the tissue type but we also have the ability to determine tissue moduli using a functional interpolant description of the MR intensity values.

During the surgical procedure, the pig brain is implanted with $1 \mathrm{~mm}$ stainless steel beads in a grid-like fashion which serve as tissue markers. A balloon catheter (filled with contrast agent) is positioned temporally. All implants are easily observable in the $\mathrm{CT}$ images. Following the procedure, incremental inflations of the balloon catheter are performed and the beads are tracked in the $\mathrm{CT}$ yielding 3-dimensional deformation maps. Following the experiment, similar boundary conditions are applied to the model producing the predicted bead displacements which are subsequently compared to the deformation maps.

\section{Results}
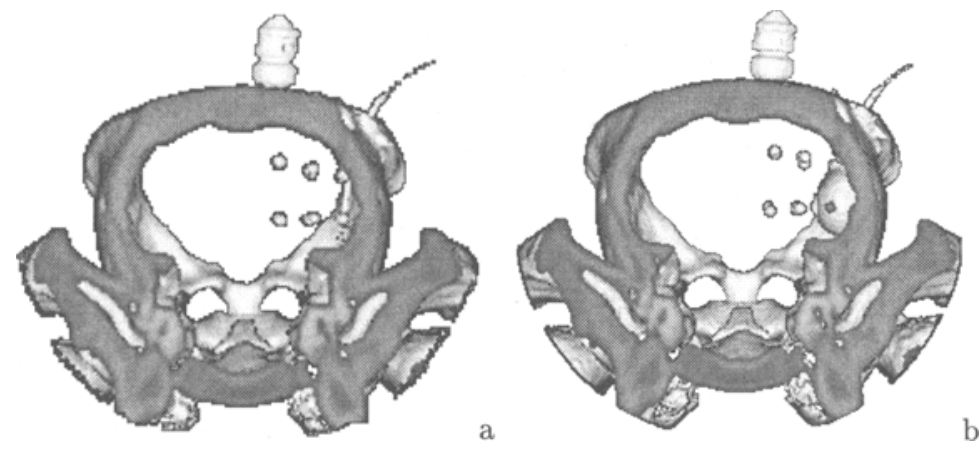

Fig. 1. Experimental model (a) baseline (b) 1 cc inflation.

The results from our in vivo porcine model can be seen in Figure 1a-b. Figure 1a-b illustrates the pig cranium with parenchymal tissue eliminated which enhances the visibility of the implants. Figure la represents the baseline position of the bead implants and the uninflated balloon catheter. The comparison figure shows movement by some beads resulting from the expanding temporally located balloon catheter. The expanded balloon surface can also be observed. The maximum displacement associated with balloon diameter for the 1cc inflation level is approximately $9.3 \mathrm{~mm}$. The average bead displacement over 15 beads is $1.52 \mathrm{~mm}$ with a maximum of $5.61 \mathrm{~mm}$.

Figures 2a illustrates a typical coronal MR slice from the pig cranium. Figure $2 \mathrm{~b}$ shows the corresponding heterogeneous slice in the discretized volume mesh. 

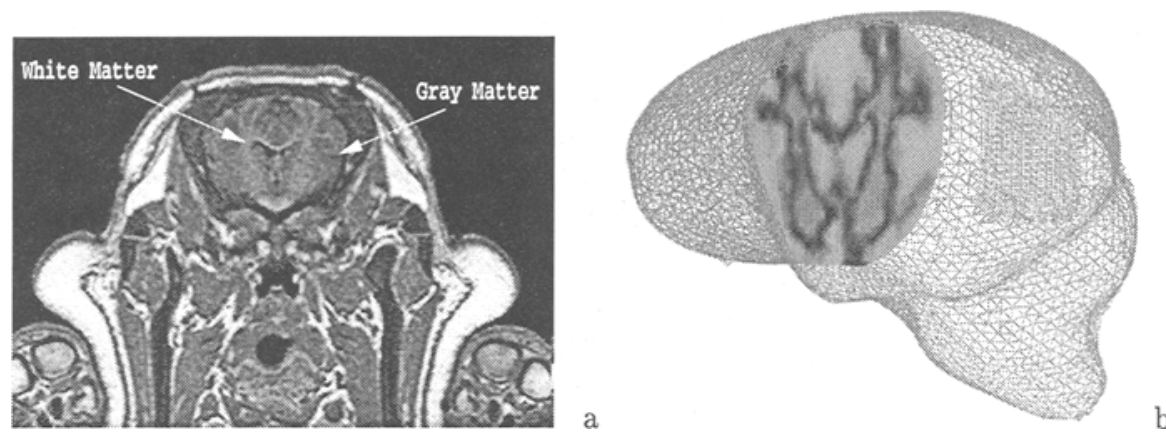

Fig. 2. Representation of heterogeneity in model with MRI slice (left) and respective heterogeneous model slice (right): (a) MRI coronal slice of pig cranium with white matter and gray matter designated (notice that white matter has a slightly higher image intensity value), (b) Volume grid boundary with equivalent rendered coronal heterogeneous slice where the dark border and its contents represent the complex material pattern of white matter surrounded by the lighter area corresponding to gray matter.

In this preliminary heterogeneous model, we have classified the continuum as one of two tissue types, white matter shown in the right image as the material enclosed by (boundary between materials is an interpolated color mapped to greyscale) the dark border and gray matter shown as the lighter material outside the border.

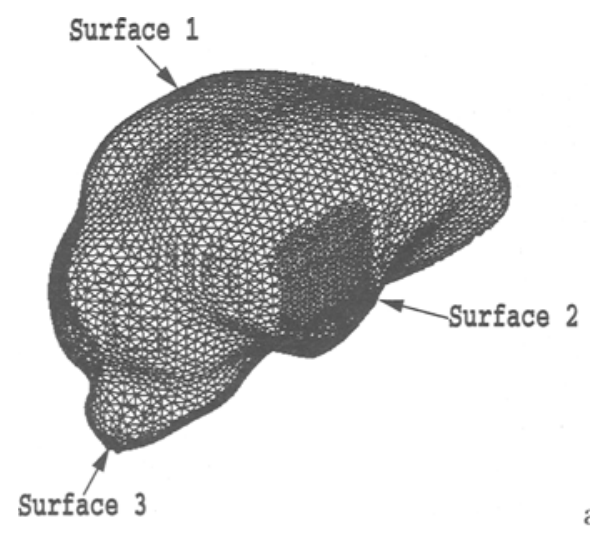

a

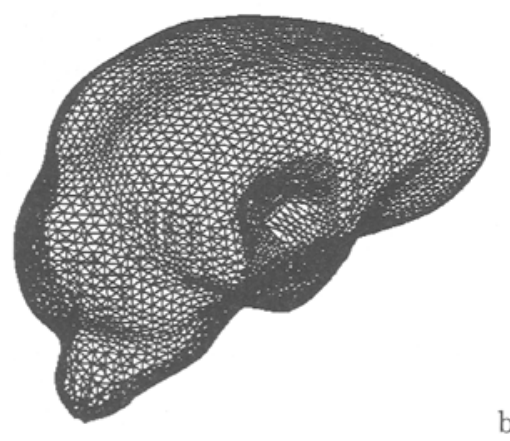

Fig. 3. (a) Boundary representation of tetrahedral grid with boundary condition surfaces designated (b) Deformed mesh from applied boundary conditions

Figure 3a displays the volumetric mesh generated by our mesh generation software with three surfaces designated where different boundary conditions are applied [17]. Surface 1 is the larger cortical surface and its boundary condition 


\begin{tabular}{|c||l|l|l|}
\hline \hline Calc. \# & $E_{w}, E_{g}(P a)$ & $\nu_{w}, \nu_{g}$ & $k_{w}, k_{g}\left(\frac{m^{3} s}{k g}\right)$ \\
\hline \hline 1 & 2100,2100 & $0.49,0.49$ & $1 \mathrm{e}-7,1 \mathrm{e}-7$ \\
\hline 2 & 2100,2100 & $0.49,0.49$ & $7.5 \mathrm{e}-12,7.5 \mathrm{e}-12$ \\
\hline 3 & 2100,2100 & $0.45,0.45$ & $1 \mathrm{e}-7,1 \mathrm{e}-7$ \\
\hline 4 & 4000,8000 & $0.47,0.47$ & $1 \mathrm{e}-7,1 \mathrm{e}-10$ \\
\hline 5 & 2700,6000 & $0.49,0.49$ & $7.5 \mathrm{e}-12,5 \mathrm{e}-12$ \\
\hline 6 & 2700,6000 & $0.49,0.49$ & $1 \mathrm{e}-7,1 \mathrm{e}-10$ \\
\hline
\end{tabular}

Table 1. Material properties for simulations with $d t=180 \mathrm{sec}$, \# steps $=3, \mathbf{u}=\mathbf{0}, p=0$.

represents a fixed, impermeable (to the fluid) surface. Surface 2 marked by the refined mesh is the location for balloon inflation. Displacement values correspond to the net balloon expansion (as measured from the CT scans) and are specified over this temporal area with an interstitial pressure of $5 \mathrm{mmHg}$. Just outside the expansion area, the surface can move freely but is impermeable to flow. Surface 3 corresponds to the brain stem and was allowed to move freely (allowing the brain to herniate) and fixed with a pressure of $0 \mathrm{mmHg}$ (gradient is formed from application area to brain stem). Under brain loading, strain-induced pressure gradients form and drainage occurs preferentially at the spinal column as the tissue is allowed to herniate. The field equations were solved on a mesh consisting of 21,732 nodes and 116,341 elements. The material properties (subscript ' $g$ ' and 'w' refer to gray and white matter, respectively) defining each calculation and the initial conditions are shown in Table 1. Figure $3 \mathrm{~b}$ displays the mesh boundary after these boundary conditions have been applied to the model. Note that the temporal surface representing the expanding balloon front has created a depression in the mesh.

Table 2 quantifies the directional and total displacement comparison between calculated and experimental bead positions. The background calculation can be viewed as a representation of the errors that would be generated by us-

\begin{tabular}{|c||l|l|l|l||l|}
\hline \hline Calc. \# & dx (mm) & dy $(\mathrm{mm})$ & dz $(\mathrm{mm})$ & D (mm) & D (\%) \\
\hline & avg. (max.) & avg. (max.) & avg. (max.) & avg. (max.) & avg. (max.) \\
\hline background & $0.39(1.17)$ & $0.26(0.78)$ & $1.35(5.47)$ & $1.52(5.61)$ & $27.2 \%(100.0 \%)$ \\
\hline 1 (Hom.) & $0.53(2.75)$ & $0.32(0.94)$ & $0.39(1.68)$ & $0.33(1.75)$ & $5.9 \%(31.1 \%)$ \\
\hline 2 (Hom.) & $0.57(2.86)$ & $0.29(0.67)$ & $0.54(1.02)$ & $0.51(1.07)$ & $9.2 \%(19.1 \%)$ \\
\hline 3 (Hom.) & $0.53(2.59)$ & $0.31(0.87)$ & $0.40(1.87)$ & $0.32(1.83)$ & $5.7 \%(32.6 \%)$ \\
\hline 4 (Het.) & $0.52(2.53)$ & $0.34(1.07)$ & $0.43(1.86)$ & $0.36(1.86)$ & $6.4 \%(33.2 \%)$ \\
\hline 5 (Het.) & $0.57(2.70)$ & $0.31(0.85)$ & $0.48(1.08)$ & $0.42(1.27)$ & $7.5 \%(22.6 \%)$ \\
\hline 6 (Het.) & $0.54(2.61)$ & $0.35(1.09)$ & $0.42(1.70)$ & $0.38(1.77)$ & $6.8 \%(31.6 \%)$ \\
\hline
\end{tabular}

Table 2. Comparison over several homogeneous (Hom.) and heterogeneous (Het.) calculations showing average (maximum) directional and total displacement error in absolute terms and the total displacement error as a percentage of the maximum bead displacement (background calculation assumes no bead movement). 
ing preoperative-based image-guided neurosurgery (i.e. assumes no movement during surgery). The first column lists the respective calculation followed by 4 columns reflecting the mean and maximum of the absolute error between experimental and calculated displacements followed by the last column relating total displacement error to the maximum experimental bead displacement as a percentage. Column 4 shows a substantial recovery of error in the ' $\mathrm{dz}$ ' component which is the direction of greatest motion for all calculations when compared to the background. Looking at the last two columns, the model-based method demonstrates an average improvement over the complete set of six different calculations of $75 \%$ (worst/best $=66 \% / 79 \%$ ) and $72 \%$ (worst $/$ best $=67 \% / 81 \%$ ) with respect to the mean and maximum total displacement relative to a surgical technique which employs no updating.

Figure 4 is representative of the physical mapping of the deformation of each bead for each directional component as well the total displacement. Each subfigure plots the displacement component versus bead number. The complete directional trajectory of a particular bead can be determined from the top two subfigures ( $\mathrm{x}$ and $\mathrm{y}$ directions) and the bottom left subfigure ( $\mathrm{z}$ direction) while the total displacement can be found in the bottom right subfigure. In each subfigure, the measured $(\mathrm{x})$ and calculated $(\mathrm{o})$ bead displacement is compared where the line segment between the two symbols represents error between predicted and actual bead movement.
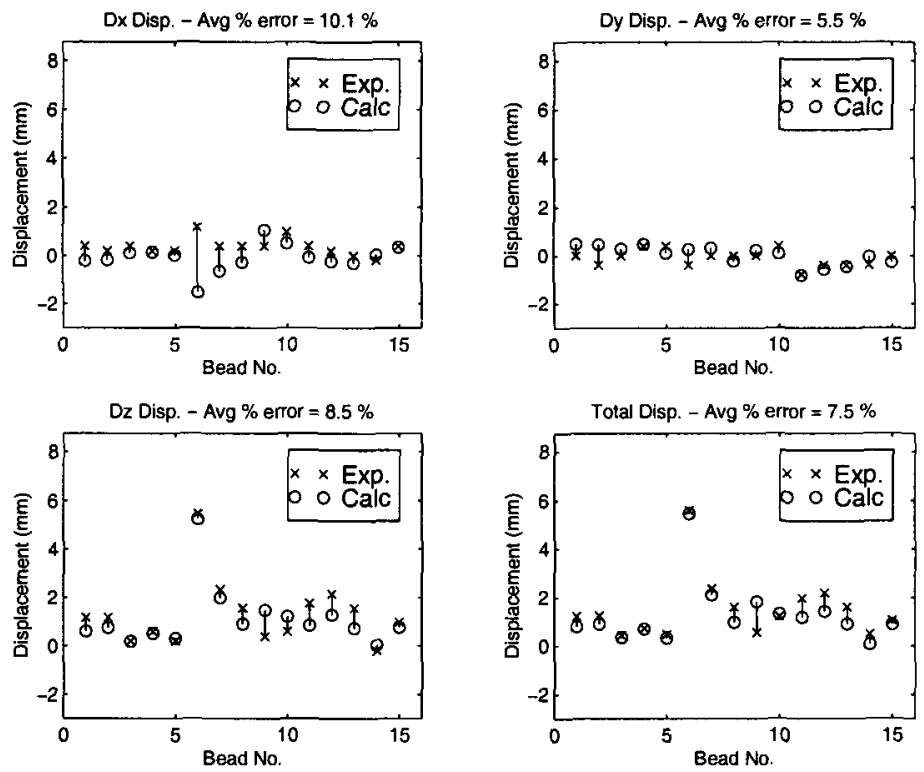

Fig. 4. Calculation \#5 (heterogeneous) comparison between measured and calculated data for directional and total displacements for $1 \mathrm{cc}$ inflation of the balloon catheter (Avg. \% error is the average error relative to the maximum bead displacement). 


\section{Discussion}

From Table 2, we see a dramatic improvement in total bead displacement for (homogeneous) calculation \#1 over the background marked by the change in absolute error from $1.52 \mathrm{~mm}$ to $0.33 \mathrm{~mm}$ in column 5. Interestingly, we observe a slightly greater decrease in error in (homogeneous) calculation \#3 to $0.32 \mathrm{~mm}$. These simulations differ only with respect to Poisson's ratio. Calculations \#1 \& \#4 as well as \#2 \& \#5 are similar in hydraulic conductivity but substantively different in Young's modulus with \#1 \& \#2 being homogeneous and \#4 \& \#5 being heterogeneous. The nominal change in total displacement between calculations \#1 \& \#4 (0.03mm) and between calculations \#2 \& \#5 (0.09 mm) suggests the impact of heterogeneity on the model may be small. However, it is important to recognize that the homogeneous property values were based on the optimal data-model match [13] whereas no such optimization has been performed with heterogeneous properties rather the values were taken from the literature.

In a similar manner we can compare these simulations by grouping them according to hydraulic conductivity. Using this criterion, we could compare homogenous calculations \#1 \& \#2 as well as heterogeneous calculations \#5 \& \#6. In the first comparison we find that calculation \#1 provides a better data-model match with respect to average total displacement whereas caclulation \#2 is the better with respect to the maximum displacement. Comparing calculations \#5 \& \#6 we find a similar trend where the simulation with smaller hydraulic conductivity yields a better result with respect to the maximum displacement error but a worse result with respect to the average. The primary difference between these simulations is the accumulation of a strain-induced pressure gradient in calculations \#2 \& \#5 due to their slow draining nature. In the course of 9 minutes, the strain-induced pressure gradients that take place in calculations \#1 \& \#6 have subsided and only the gradient from the deformation source to brain stem remains ( $5 \mathrm{mmHg}$ ). However, \#2 \& \#5's strain-induced pressure gradients are on the order of $35 \mathrm{mmHg}$ and act as a distributed body force which produces more deformation.

In Table 2, we have provided a background calculation. This calculation can be viewed as a representation of the loss in accuracy due to using preoperativebased image-guided surgery; it assumes that no movement has occurred from the initial image baseline which is exactly the same assumption which underpins preoperative-based image-guided neurosurgery. Using this measure as a background against which to compare subsequent model calculations provides insight into the recovery of accuracy that can be attained using our model-guided approach. It also suggests the degree to which each component of the model influences the accuracy outcome. Examining the averages in the last column of Table 2 and comparing the percentages to the background, we can see that our calculations indicate that elasticity accounts for approximately $63 \%-79 \%$ of the deformation, pressure gradients account for $5 \%-12 \%$ and heterogeneity accounts for $0.5 \%-2 \%$. This breakdown ranks the importance of each component of the model and the approximate recovery of accuracy that might be expected using our approach. 
Analyzing Figure 4 yields an indication of how well the model can track bead displacement. The majority of displacement in this case occurs along the $\mathbf{z}$ axis which is the direction approximately normal to the expanding balloon front. Overall, we can see that the directional trends are quantitatively captured and there is excellent agreement between experimental and calculated total displacement. Given the simplicity and approximate nature of the boundary conditions, the results presented here are very encouraging for the future of model-updated image-guided stereotactic neurosurgery.

\section{Conclusions}

In previous results, we have shown that a 3D consolidation model is capable of predicting the total average displacement to within $15 \%$ of measured values for an intraparenchymally located expanding mass [7], [13] and $6.6 \%$ for a temporally located mass [8] within a porcine brain. In this paper, we have demonstrated that we are able to predict a total average displacement within $5.7 \%$ for a temporally expanding mass using a more complex model which includes pressure gradients, tissue property heterogeneity and better mesh resolution. In the analysis of several calculations, we have found that the effects of heterogeneity are relatively minor, accounting for a $0.5 \%-2 \%$ change in results, while effects from elasticity and pressure gradients account for $63 \%-79 \%$ and $5 \%-12 \%$, respectively. However, it is important to recognize that we have not explored the full range of possible combinations of heterogeneous tissue properties in order to best fit the experimental data. Further, there is no consensus on the exact values for these properties in gray versus white matter. Hence, we may have underestimated the importance of tissue heterogeneity at this point and further study is warranted. Nonetheless, our approach has shown an ability to capture nearly $75 \%$ of the tissue registration inaccuracy generated by the equivalent of preoperative-based image-guided neurosurgery. The remaining $25 \%$ has likely been lost for several reasons of which the most significant may be the inability to adequately describe the expanding balloon front creating error associated with bead directionality. We are presently in the process of designing a piston-like delivery system for more controlled applications of deformation. Undoubtedly, there is additional accuracy loss due to large deformation, non-linearities and experimental error (i.e. coregistration and bead fixation problems); but, overall the results shown here, as well as previously, are very encouraging for this model-updating approach.

Acknowledgments: This work was supported by National Institutes of Health grant R01-NS33900 awarded by the National Institute of Neurological Disorders and Stroke. ANALYZE software was provided in collaboration with the Mayo Foundation.

\section{References}

1. D. W. Roberts, J. W. Strohbehn, J. F. Hatch, W. Murray, and H. Kettenberger, ' A frameless stereotactic integration of computerized tomographic imaging and the operating microscope', J. Neurosurg., vol. 65, pp. 545-549, 1986. 
2. T. Peters, B. Davey, P. Munger, R. Comeau, A. Evans, and A. Olivier, 'Threedimensional multimodal image-guidance for neurosurgery', IEEE Trans. Med. Imaging, vol. 15, pp. 121-128, 1996.

3. D. W. Roberts, A. Hartov, F.E. Kennedy, M. I. Miga, K. D. Paulsen, 'Intraoperative brain shift and deformation: a quantitative clinical analysis of cortical displacements in 28 cases', Neurosurgery, (in press), 1998.

4. D. L. G. Hill, C. R. Maurer, M. Y. Wang, R. J. Maciunas, J. A. Barwise, J. M. Fitzpatrick, 'Estimation of intraoperative brain surface movement', Proc. CVRMedMRCAS'97, ed. J. Troccaz, E. Grimson, R. Mosges, Springer Lecture Notes in Computer Science, vol. 1205, pp. 449-458, 1997.

5. H. Dickhaus, K. Ganser, A. Staubert, M. M. Bonsanto, C. R. Wirtz, V. M. Tronnier, and S. Kunze, 'Quantification of brain shift effects by mr-imaging', Proc. $A n$. Int. Conf. IEEE Eng. Med. Biology Soc., 1997.

6. M. I. Miga, K. D. Paulsen, F. E. Kennedy, P. J. Hoopes, A. Hartov, and D. W. Roberts, 'A 3D brain deformation model experiencing comparable surgical loads', Proc. 19th An. Int. Conf. IEEE Eng. Med. Biology Soc., 773-776, 1997.

7. K. D. Paulsen, M. I. Miga, F. E. Kennedy, P. J. Hoopes, A. Hartov, and D. W. Roberts, 'A computational model for tracking subsurface tissue deformation during stereotactic neurosurgery', IEEE Transactions on Biomedical Engineering, (in press), (1998).

8. M. I. Miga, K. D. Paulsen, F. E. Kennedy, P. J. Hoopes, A. Hartov, and D. W. Roberts, 'Quantification of a 3D brain deformation model experiencing a temporal mass expansion', Proceedings of the 24th IEEE Northeast Bioengineering Conference, pp. 68-71, 1998.

9. M. Biot, 'General theory of three dimensional consolidation', J. Appl. Phys., vol. 12, pp. 155-164, 1941.

10. M. I. Miga, K. D. Paulsen, F. E. Kennedy, 'Von Neumann stability analysis of Biot's general two-dimensional theory of consolidation', Int. J. of Num. Methods in Eng., (in press), 1998.

11. E. K. Walsh, and A. Schettini, 'Calculation of brain elastic parameters in vivo', Am. J. Physiol., 247, R693-R700, 1984.

12. R. Mathupillai, P. J. Rossman, D. J. Lomas, J. F. Greenleaf, S. J. Riederer, and R. L. Ehman, 'Magnetic resonance elastography by direct visualization of propagating acoustic strain waves', Science, vol. 269, pp. 1854-1857, 1995.

13. M. I. Miga, K. D. Paulsen, F. E. Kennedy, P. J. Hoopes, A. Hartov, and D. W. Roberts, 'Modeling Surgical Loads to Account for Subsurface Tissue Deformation During Stereotactic Neurosurgery', IEEE SPIE Proceedings of Laser-Tissue Interaction IX, Part B: Soft-tissue Modeling, vol. 3254, pp. 501-511, 1998.

14. T. Nagashima, T. Shirakuni, and SI. Rapoport, 'A two-dimensional, finite element analysis of vasogenic brain edema,' Neurol. Med. Chir., vol. 30, pp. 1-9, 1990.

15. P. J. Basser, 'Interstitial pressure, volume, and flow during infusion into brain tissue', Microvasc. Res., vol. 44, pp. 143-165, 1992.

16. C. Montani, R. Scateni, and R. Scopigno, 'Discretized Marching Cubes Visualization 1994', Conf. Proc., Washington, 1994.

17. J. M. Sullivan Jr., G. Charron, and K. D. Paulsen, 'A three dimensional mesh generator for arbitrary multiple material domains, Finite Element Analysis and Design, vol. 25, pp. 219-241, 1997. 